\title{
Empoderamiento y relación con la satisfacción laboral en personas con movilidad reducida
}

\section{Empowerment and its relationship with job satisfaction in people with reduced mobility}

\begin{abstract}
Resumen
En este estudio se explora la posible asociación entre diferentes perfiles de empoderamiento y la satisfacción laboral de una muestra de trabajadores con movilidad reducida. La Escala de Satisfacción Laboral General (1979) y la Escala de Empoderamiento (1997), fueron administradas a 67 trabajadores con movilidad reducida, con edades comprendidas entre 26 y 52 años $(M=31,22, D . T .=1,35)$. Los resultados indicaron que los participantes presentaron niveles moderados de satisfacción laboral, observándose que los trabajadores con mayor nivel de empoderamiento indicaron mayor satisfacción laboral. Asimismo, el índice de correlación mostró una alta asociación entre satisfacción laboral y empoderamiento. Esto sugiere que el contexto laboral es uno de los ámbitos prioritarios para fomentar el empoderamiento de los trabajadores con movilidad reducida y con ello mejorar su bienestar laboral.
\end{abstract}

\section{Palabras clave}

Discapacidad, integración laboral, fortaleza, calidad de vida.

\begin{abstract}
The present study is aimed to analyse the possible association between different profiles of empowerment and job satisfaction by using a sample of workers with reduced mobility. The Overall Job Satisfaction Scale (1979) and the Empowerment Scale (1997) were administered to 67 people with reduced mobility, between 26 to 52 years of age $(M=31.22$; $S D=1.35)$. Results showed moderated levels of job satisfaction among participants, and that such satisfaction was even higher in workers with a high level of empowerment. Likewise, the correlation index showed a high association between job satisfaction and empowerment. This suggests that the labour context is one of the priority areas to promote the empowerment of workers with reduced mobility, and therefore, to improve their work well-being.
\end{abstract}

\section{Keywords}

Disability, labour integration, strength, quality of life.

\author{
Raquel Suriá Martínez \\ $<$ raquel.suria@ua.es>
}

Universidad de Alicante. España

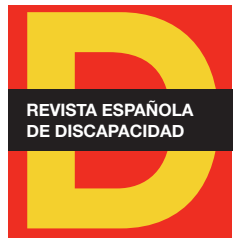

Para citar:

Suriá, R. (2020). "Empoderamiento y relación con la satisfacción laboral en personas con movilidad reducida". Revista Española de Discapacidad, 8(l), pp. 145-157.

Doi: <https://doi.org/10.5569/23405104.08.01.08>

Fecha de recepción: 24-05-2018 Fecha de aceptación: 09-01-2020 


\section{Introducción}

En los últimos años, el interés por fomentar la inserción laboral de las personas con discapacidad se ha incrementado desde diferentes ámbitos. Según indica el informe de la OCDE (2010), ha habido un cambio sustancial laboral en este sector, ya que ha pasado de utilizar políticas pasivas basadas en el subsidio y las pensiones, a una situación donde el objetivo, es buscar la mayor estabilidad del colectivo con discapacidad dentro del mercado laboral (Flores et al., 2011; Sá-Fernandes et al., 2018), debiéndose esto, en gran parte, a la implicación de la Organización Internacional del Trabajo (OIT 2013), que aprobó convenios y recomendaciones a través de la promoción de las normas laborales, actividades de sensibilización y estrategias de inclusión en programas sobre la formación y el empleo con el objetivo de lograr el respeto a la igualdad de oportunidades, la inclusión e integración social, el derecho al trabajo y las condiciones laborales dignas y satisfactorias una vez que este colectivo se encuentra ejerciendo sus labores (Flores et al., 2011; Kocman y Weber, 2018; Pallisera y Rius, 2007).

En este sentido, si bien es importante lograr que las personas con discapacidad tengan la oportunidad de optar por un empleo, es fundamental que sus condiciones laborales sean igual de satisfactorias que las del resto de trabajadores (Alcover de la Hera y Pérez Torres, 2011; Durá y Salaberría, 2011).

Uno de los indicadores más relevantes estudiados en el contexto laboral en trabajadores con discapacidad es la satisfacción laboral. Este constructo ha sido definido por diferentes autores como la respuesta evaluativa, afectiva y conductual hacia el trabajo que se desempeña (Pecino-Medina et al., 2015; Veloso-Besio et al., 2015).

Por su parte, Judgel et al. (2012) intentan dar un sentido más exacto a la definición y consideran otros aspectos más concretos del trabajo, como puede ser la retribución, el trato y fundamentalmente, el apoyo social ofrecido por los compañeros, de la organización, de la empresa, etc.

En este sentido, la evidencia empírica sobre la satisfacción laboral en trabajadores con discapacidad incide en que las principales fuentes de apoyo social en el contexto laboral, pueden ser los supervisores (Beehr et al., 2000), los compañeros de trabajo (Kocman y Weber, 2018), así como la organización (Flores et al., 2010).

Entre las formas de apoyo más valoradas por este colectivo de trabajadores destacan el instrumental (por ejemplo, el ayudar en el cumplimiento de las tareas) y el emocional, como pueden ser los comportamientos de empatía, cuidado y escucha (Beehr et al., 2000). Del mismo modo, el apoyo organizacional que perciben los trabajadores les permite que valoren la ayuda que les ofrece la organización, la forma como se aprecia su contribución a las metas y el cuidado que le ofrece la empresa (Eisenberger, et al., 2001). La literatura sobre calidad de vida de las personas con discapacidad e incorporación laboral señalan que el apoyo organizacional influye sobre el compromiso y el bienestar de los empleados (Kocman y Weber, 2018; Riaño-Galán et al., 2016). Esto se debe a que favorece el sentido de reciprocidad, satisface las necesidades sociales de autoestima, afiliación e identidad social que hace sentir a los trabajadores como una parte importante de la organización (Eisenberger, et al., 2001; Riaño-Galán et al., 2016). A su vez, esto se relacionaría con el apego que percibe el trabajador por la organización, puesto que favorece un mayor compromiso en el que se asumen como propios los logros y, por tanto, con una mayor satisfacción laboral (Rhoades y Eisenberger, 2002). 
En relación a esto, otros autores como Moreno et al. (2009) o Peralta et al. (2016), conceptualizan la satisfacción laboral como una actitud que asume la persona ante su trabajo y que se refleja en actitudes, sentimientos, estados de ánimo y comportamientos en relación a su actividad laboral, por lo que en esta definición, además de las variables externas a la persona (condiciones del trabajo, apoyos, entorno laboral etc.,), cobran relevancia otras variables de la personalidad como por ejemplo, la inteligencia emocional, estilos de afrontamiento, etc., (Peralta et al., 2016).

Una de las variables que está cobrando un papel importante en la calidad de vida de las personas con discapacidad es el empoderamiento (Rappaport, 1984). Literalmente, puede ser traducido como "empoderar", es decir, dar poder, dotar de poder, pero el término empowerment se traduce generalmente, no como empoderar, sino como potenciación o fortalecimiento (Musitu y Buelga, 2004). Así, este concepto, hace referencia al potencial de cada persona para alcanzar objetivos y metas definidos por ellas mismas, abordando de este modo la vida, en términos de oportunidades personales y sociales (Rappaport, 1984; Zimmerman, 2000).

En este sentido, en el documento del Programa de Las Naciones Unidas para el Desarrollo (PNUD) presentado en la Conferencia Internacional para el Desarrollo Humano (1997), se observa el empoderamiento como el proceso mediante el cual los individuos obtienen control de sus decisiones y acciones relacionadas con su salud; expresan sus necesidades y se movilizan para obtener mayor acción política, social y cultural para responder a dichas necesidades, a la vez que se involucran en la toma de decisiones para el mejoramiento de su salud y la de la comunidad. Por tanto, el empoderamiento se asocia en primer lugar, con el poder, cambiando las relaciones de poder en favor de aquellos que con anterioridad tenían escasa autoridad sobre sus propias vidas, reflejándose en este caso el poder como capacidad humana, que permite, una mayor autoestima y un proceso de transformación de la propia conciencia puede vencer multitud de barreras (Navarro-Pérez y Martínez-Muñoz, 2014).

A partir de esta descripción, la literatura sobre este constructo (Albrecht et al., 2001; Barnes et al., 1997; Bejerholm y Björkman, 2011; Segado, 2011); por citar algunos de los más representativos), está de acuerdo en señalar que el empoderamiento no es un proceso lineal, con un principio y un fin claramente definido y común para todos los individuos, sino que es un proceso que se experimenta de manera diferenciada y de alguna manera única por cada individuo, y se define y desarrolla en función de la historia personal y del contexto, por tanto, puede ocurrir por efectos de experiencias diversas, tales como procesos educativos, organizativos, laborales, etc. Es en este contexto, en el laboral y oganizacional, en el que empoderamiento incluye un compendio de procesos y estructuras que incrementan la participación de sus miembros y mejoran el logro de resultados para la organización (Rico-Picó et al., 2016). Del mismo modo, a nivel comunitario este constructo se puede relacionar con la identificación organizacional como un mediador de influencia, conformada por componentes cognitivos y emocionales (Koberg et al., 1999). Éstos hacen referencia a compartir intereses, características esenciales y sentimientos positivos, principalmente. Así pues, se ha visto que los antecedentes del empoderamiento, como la percepción de respaldo por parte de la organización que tiene el trabajador o la confianza en el supervisor, hacen también que la persona sienta un mayor nivel de identificación grupal. Finalmente, también se ha visto relacionada con algunas de las consecuencias que se le atribuyen al empoderamiento, por ejemplo, una correlación positiva con la satisfacción y el compromiso organizacional y de todos los trabajadores (Ríos Manríquez et al., 2010).

En este sentido, el estudio sobre el empoderamiento, ha sido utilizado en diversas disciplinas con el propósito de comprender y fomentar la integración de colectivos en desventaja o vulnerabilidad social, como 
en minorías étnicas (Bertely, 2003; Weissenberg, 1999), en mujeres (Gallway y Bernasek, 2004; Röger et al., 2011), y en personas con discapacidad (Saavedra y Villalta, 2008; Suriá, 2017).Si se tiene en cuenta la dificultad de inserción laboral de las personas con discapacidad (Baldridge y Swift, 2013), y posteriormente, los diferentes obstáculos a los que se pueden ver sometidos estos trabajadores en el día a día de su desarrollo profesional, como por ejemplo, bajo apoyo social, elevadas demandas psicológicas, alta inseguridad laboral o poca capacidad de decisión (Moore et al., 2011), es cuando cobra sentido el empoderamiento, pues ello puede permitir potenciar la autodeterminación, la autonomía, la toma de decisiones y, en general, la calidad de vida (Alcover et al., 2017).

Al atender a la literatura sobre el empoderamiento y su relación con la satisfacción laboral y aunque si se ha estudiado la relación de la satisfacción laboral con otras variables influyentes, como por ejemplo la inteligencia emocional (Landa et al., 2006), el engagement (Durán et al., 2005), o el liderazgo (Cuadra y Veloso, 2007), desde este estudio no se han encontrado trabajos que analicen la relación de estos dos constructos en personas con diversidad funcional, y concretamente en trabajadores con movilidad reducida.

Por tanto, en este estudio se pretende examinar la posible asociación entre diferentes perfiles de empoderamiento y la satisfacción laboral de una muestra de trabajadores con movilidad reducida, a la vez que comprobar que otras variables relacionadas con el perfil sociodemográfico y laboral de los trabajadores influyen en el nivel de satisfacción laboral de este colectivo de trabajadores. Este objetivo se desglosa en los siguientes objetivos específicos:

1. El primer objetivo examina el perfil (alto/medio/bajo) de empoderamiento en una muestra de trabajadores con movilidad reducida. En este sentido esperamos que:

H1. Existan diferentes perfiles de empoderamiento entre la muestra de trabajadores.

2. El siguiente objetivo, examina si existen diferencias estadísticamente significativas en la satisfacción laboral en función de los perfiles de empoderamiento de los participantes obtenidos en el primer objetivo. En concreto, esperamos que:

H.2. Existan diferencias estadísticamente significativas en el nivel de satisfacción laboral, observándose mayores niveles de satisfacción laboral en el grupo con puntuaciones elevadas en empoderamiento.

3. Finalmente, el último objetivo explora si existe asociación entre la satisfacción laboral y el perfil sociodemográfico (sexo, edad, grado de discapacidad) y laboral (tipo de trabajo, de contrato y antigüedad laboral) de los trabajadores. Así, esperamos que:

H3. Exista relación entre la satisfacción laboral y el perfil sociodemográfico y laboral de los participantes.

\section{Método}

\subsection{Participantes}

Se trata de un diseño transversal conformado por una muestra intencional -por motivos de accesibilidadde personas con movilidad reducida de la asociación de ASPAYM (Asociación de Parapléjicos y Personas 
con Gran Discapacidad Física) en la Comunidad Valenciana, formada por 900 socios. La población elegible estuvo constituida inicialmente por los 142 socios mayores de 18 años con movilidad reducida que acudían a alguna de las reuniones convocadas periódicamente por la asociación. De ellos, 92 socios (la muestra final de estudio), se encontraban en situación laboral activa, ocupando empleos de modalidad ordinaria, siendo 67 de estos los que accedieron a participar tras asistir a las reuniones presenciales en las que desde la investigación. Las características sociodemográficas de la composición de la muestra se indican en la tabla 1:

\begin{tabular}{|c|c|c|c|}
\hline \multicolumn{2}{|l|}{ Perfil sociodemográfico } & \multirow{2}{*}{$\frac{\mathbf{N}}{23}$} & \multirow{2}{*}{$\begin{array}{c}\% \\
34,3\end{array}$} \\
\hline Coro & Mujer & & \\
\hline UEd & Varón & 44 & 65,7 \\
\hline \multirow{3}{*}{ Edad } & Entre 26 y 35 & 33 & 49,3 \\
\hline & Entre 36 y 45 & 18 & 26,9 \\
\hline & Más de 45 & 16 & 23,9 \\
\hline \multirow{3}{*}{ Grado de discapacidad } & Más del $75 \%$ & 27 & 40,3 \\
\hline & Entre 25 y $50 \%$ & 20 & 29,9 \\
\hline & Entre 51 y $75 \%$ & 20 & 29,9 \\
\hline \multirow{4}{*}{ Tipo de trabajo } & Alimentación & 26 & 38,8 \\
\hline & Confección & 14 & 20,9 \\
\hline & Hostelería & 11 & 16,4 \\
\hline & Limpieza & 16 & 23,9 \\
\hline \multirow{3}{*}{ Tipo de contrato } & Interino & 19 & 28,4 \\
\hline & Temporal & 21 & 31,3 \\
\hline & Fijo & 27 & 40,3 \\
\hline \multirow{4}{*}{ Antigüedad laboral } & Menos de 5 años & 14 & 20,9 \\
\hline & Entre 5 y 10 años & 32 & 47,8 \\
\hline & Más de 10 años & 21 & 31,3 \\
\hline & Total & 67 & 100,0 \\
\hline
\end{tabular}

Fuente: elaboración propia.

\subsection{Instrumentos}

- Escala de empoderamiento de Rogers et al. (1997). La escala está formada por un total de 28 ítems, con respuesta tipo Likert de 4 puntos ( $0=$ nada de acuerdo, 3=muy de acuerdo). La información solicitada recoge aspectos en relación a la percepción del propio participante en función de la capacidad para la toma de decisiones (ej. "Enfadarse por algo nunca sirve de ayuda", "Soy capaz de hacer cosas tan bien como la mayoría" ó "Tengo una actitud positiva acerca de mí mismo/a"). La puntuación máxima es de 84 puntos, no obstante, para establecer una medida de corte se siguió el criterio de San Martín y Pardo 
(1989), concretándose tres niveles, separándolos en tres terciles. (nivel bajo= de 0 a 27, nivel medio= de 28 a 56 y nivel alto= de 57 a 84). Se eligió esta escala siguiendo varios criterios: por su fácil aplicación, por estar validado para población joven y adulta y finalmente, por las propiedades psicométricas que muestra la versión original (Rogers et al., 1997). Así, el estudio original indicó una fiabilidad del $86 \%$ $(a=, 86)$. Del mismo modo, la validez de la escala original mostró una varianza explicada del 53,9\%, quedando definido por cinco factores: Autoestima-Autoeficacia (explica el $24,5 \%$ de la varianza total); Poder/Impotencia (explica el $12,4 \%$ de la varianza total); Autonomía (explica el 7,6\% de la varianza total); Optimismo-Control sobre el futuro (explica el 5,4\% de la varianza total); Ira apropiada (explica el $4 \%$ de la varianza total).

- Para medir Satisfacción Laboral se utilizó la Escala de Satisfacción Laboral General, desarrollada por Warr et al., (1979) y traducida y adaptada al español por Pérez-Bilbao y Fidalgo (1995). Esta escala es aditiva, en la cual la puntuación total se obtiene de la suma de los posicionamientos de encuestado en cada uno de los quince ítems, asignando un valor de 1 a Muy insatisfecho y correlativamente hasta asignar un valor de 7 a Muy Satisfecho (ej.: "Condiciones físicas del trabajo!, "Compañeros" ó "Posibilidades de promocionar"). De este modo, tal como queda establecido por los autores de la escala, la puntuación total del instrumento oscila entre 15 y 105, de manera que una mayor puntuación refleja una mayor satisfacción general. A su vez, la escala queda dividida en tres rangos, indicándose una satisfacción laboral baja las puntuaciones inferiores a 45; de 45 a 74 satisfacción media y de 75 a 105 satisfacción elevada.

Este instrumento fue creado a partir de detectarse la necesidad de escalas cortas y robustas que pudieran ser fácilmente completadas por todo tipo de trabajador con independencia de su formación. Se ha escogido este instrumento por ser breve (requiere aproximadamente 15 minutos su cumplimentación), específico (mide aserción y/o habilidades sociales), construido, validado y tipificado para población española y con unas adecuadas propiedades psicométricas (validez y fiabilidad). La fiabilidad obtenida por sus autores (Warr et al., 1979), y estimada a través del indicador alfa de Cronbach, va desde .85 a .88. Esta escala ha sido utilizada previamente en estudios de este país, contando con propiedades psicométricas adecuadas de validez estructural y fiabilidad (Cuadra y Veloso, 2007).

\subsection{Procedimiento}

La selección de la muestra de trabajadores fue por conveniencia. Así, se acudió a la asociación ASPAYM CV, destinada al colectivo de personas con movilidad reducida. El contacto inicial fue con los directivos de la asociación, a ellos se le explico el estudio y nos invitaron a acudir a sus reuniones presenciales para explicarles directamente a los socios el objetivo del estudio y proponerles su participación voluntaria en el estudio. Los cuestionarios utilizados para recabar la información se administraron a través de un enlace creado en la plataforma Google, el cual fue creado específicamente para el estudio, los participantes otorgaron su consentimiento por escrito. La aplicación del cuestionario se adaptó a las condiciones de cada participante. El tiempo estimado de aplicación fue aproximadamente de 15 minutos. La recogida de datos se llevó a cabo entre marzo del 2016 y enero del 2017. 


\subsection{Análisis estadístico}

Para definir los perfiles de empoderamiento se siguió el criterio de concretar tres niveles, separándolos en tres terciles (San Martín y Pardo, 1989). Para comprobar si existían diferencias a nivel estadísticamente significativo se utilizó la prueba no paramétrica de Kruskal Wallis. Posteriormente los contrastes post hoc se realizaron con la prueba $U$ de Mann-Whitney, y el ajuste de Bonferroni. Se emplearon pruebas no paramétricas por el reducido número de participantes y porque las variables no cumplían el supuesto de homocedasticidad de varianza.

Finalmente, con el objetivo de deslindar la asociación de la satisfacción laboral con las variables independientes posiblemente relacionadas (grado de discapacidad, sexo, edad, tipo de trabajo, tipo de contrato, antigüedad laboral y empoderamiento), se calculó el coeficiente de correlación de Spearman. Los datos se analizaron por medio del paquete estadístico SPSS versión 20.0.

\section{Resultados}

Objetivo 1. Examinar el perfil (alto/medio/bajo) de empoderamiento en una muestra de trabajadores con discapacidad motora.

Se conformaron 3 grupos de trabajadores en función de su nivel de empoderamiento, un grupo formado por 28 participantes (41,8\%), con bajos niveles de empoderamiento (Grupo 1), un grupo con empoderamiento medio (Grupo 2) formado por 21 participantes (31,3\%), y un tercer grupo con alto empoderamiento (Grupo $3)$, conformado por 18 trabajadores $(26,9 \%)$.

\section{Figura 1. Perfiles de empoderamiento}

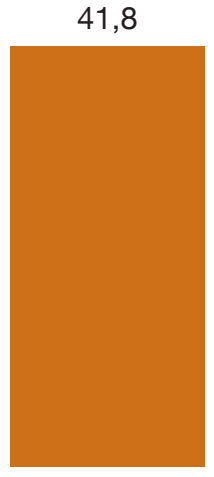

Nivel bajo
31,3

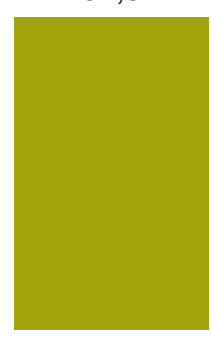

Nivel medio
26,9

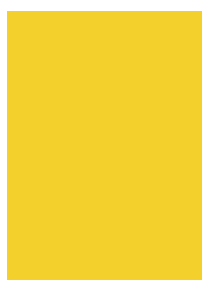

Nivel elevado

Fuente: elaboración propia. 
2.- Segundo objetivo, examinar si existen diferencias estadísticamente significativas en la satisfacción laboral en función de los perfiles de empoderamiento de los participantes.

En general, se observó que los participantes presentaron niveles medios de satisfacción laboral en la escala global. Sin embargo, al atender a las puntuaciones medias de la escala total en función de los tres perfiles de empoderamiento obtenidos en el objetivo 1 esto es, bajo, medio y elevado, se observaron diferencias estadísticamente significativas en los tres perfiles $\left[\chi_{(2, N=67)}=36,62, p<, 001\right]$, encontrándose que el Grupo 3 indicó índices medios superiores al Grupo 2 y al Grupo 1. Asimismo, el Grupo 2 mostró puntuaciones medias más elevadas que el Grupo 1.

\begin{tabular}{|c|c|c|c|c|}
\hline Perfil de empoderamiento & $N$ & $M$ & D.T. & $\begin{array}{c}\text { Sig. Estadística } \\
\chi^{2}(2,67)\end{array}$ \\
\hline Nivel bajo & 28 & 38,14 & 9,38 & \multirow{4}{*}{$36,62^{\star *}$} \\
\hline Nivel medio & 21 & 48,66 & 9,17 & \\
\hline Nivel elevado & 18 & 72,50 & 15,48 & \\
\hline Total & 67 & 50,67 & 17,92 & \\
\hline \multicolumn{5}{|c|}{ Prueba de Kruskal Wallis } \\
\hline
\end{tabular}

Fuente: elaboración propia.

3. Tercer objetivo averigua la relación entre satisfacción laboral y el perfil sociodemográfico de los trabajadores (sexo, edad y grado de discapacidad), así como entre satisfacción laboral y perfil laboral (tipo de trabajo, tipo de contrato y antigüedad laboral).

En referencia a la relación entre satisfacción laboral y el perfil de los participantes (Tabla 3), los resultados indicaron una asociación positiva entre la edad de los trabajadores y la satisfacción laboral (49,2\%). Del mismo modo, al examinar la relación entre satisfacción laboral y variables laborales, se encontró relación positiva entre tipo de trabajo y satisfacción laboral $(41,2 \%)$ y de ésta con la antigüedad laboral $(47,6 \%)$.

\begin{tabular}{|c|c|c|c|}
\hline & Edad & Sexo & Grado discapacidad \\
\hline \multirow[t]{2}{*}{ Satisfacción laboral } &, $492^{* *}$ & ,122 &, 137 \\
\hline & Tipo trabajo & Tipo contrato & Antigüedad laboral \\
\hline Satisfacción laboral & .15 &, $412^{\star \star}$ &, $476^{\star \star}$ \\
\hline
\end{tabular}

**. La correlación es significativa en el nivel 0,01 (bilateral)

Fuente: elaboración propia. 


\section{Discusión}

En general, los resultados de la escala reflejan que el grado de empoderamiento de la mayoría de los participantes se encuentra moderadamente alto. Estos resultados respaldan los obtenidos por otros autores que encontraron asociación entre personas con discapacidad y niveles elevados de empoderamiento, observándose en la mayoría, un alto nivel de autoeficacia y sentimientos de control de sus vidas (Corrigan, 2002; Corrigan y Gelb, 2006; Suriá, 2017). Esto facilitará en gran medida su satisfacción en general, reflejándose por tanto en el ámbito laboral.

Estos resultados confirman la primera hipótesis y respalda los resultados del siguiente objetivo, explorar si existen diferencias en la satisfacción laboral según el perfil de empoderamiento de los trabajadores. Así, se observa que el perfil de trabajadores que puntúan alto en su nivel de satisfacción laboral es el del grupo de trabajadores con puntuaciones elevadas en empoderamiento. Esto indica que se cumple la segunda hipótesis del estudio, existen diferencias estadísticamente significativas en el nivel de satisfacción laboral, observándose mayores niveles de satisfacción laboral en el grupo de alto empoderamiento. Esto confirma la segunda hipótesis del estudio. Sin embargo, nuestros resultados se verían parcialmente respaldados con otros estudios. Así, en diferentes estudios se ha encontrado un nivel de satisfacción con el trabajo medioalto en trabajadores con discapacidad (Flores et al., 2010b) del mismo modo, Hansen y Nielsen (2008), también se obtienen resultados de alta satisfacción en personas con discapacidad, en comparación con personas sin discapacidad. En todos ellos, parece que el motor para experimentar satisfacción laboral es el mero hecho de obtener un empleo (Cuadra y Veloso, 2007) y no como resultado de su bienestar respecto a otras dimensiones laborales y personales.

En este sentido, nuestros resultados reflejan que es el grupo con empoderamiento el que resalta por sus elevadas puntuaciones en satisfacción laboral, por tanto, sería necesario un nivel adecuado de esta capacidad para la satisfacción laboral. Así, en las líneas anteriores se ha definido que el empoderamiento se asociaría con un cambio en las relaciones de poder que permitiría una mayor autoestima y un proceso de transformación de la propia conciencia para vencer multitud de barreras (Navarro-Pérez y Martínez-Muñoz, 2014). Al tiempo incluye un compendio de procesos y estructuras que incrementan la participación de sus miembros y mejoran el logro de resultados para la organización (Rico-Picó et al., 2016) así como para compartir intereses y sentimientos positivos que favorecen que la persona sienta un mayor nivel de identificación grupal y personal, lo que conllevaría a una satisfacción en sus actividades diarias y por tanto en el contexto laboral.

Con respecto a los resultados del tercer objetivo, analizar la relación entre la satisfacción laboral y las diferentes variables de estudio, los datos reflejan que la satisfacción laboral se asocia de manera positiva con el empoderamiento. Estos resultados van en la misma dirección de la de otros estudios (Seibert et al., 2004; Seibert et al., 2011), los cuales demostraron que el empoderamiento se correlaciona positivamente con una mayor satisfacción, rendimiento y desempeño en el trabajo. En este sentido, y al centrarnos de forma específica en el término empoderamiento, éste hace alusión al mecanismo por el cual las personas, las organizaciones y las comunidades ganan control, maestría o dominio sobre sus propios destinos (Rappaport, 1984). Así, por ejemplo, Bejerholm y Björkman, (2011) lo definen como un proceso por el cual los individuos aprenden a ver la correspondencia entre sus metas y la sensación de saber cómo alcanzarlas, así como la asociación entre el esfuerzo realizado y el resultado que obtienen. Por tanto, el sentirse empoderado puede 
actuar como un mediador al estar formado por las cogniciones referentes a la evaluación de las experiencias clave para que se produzca la satisfacción en el ámbito laboral (Wang y Lee, 2009).

Con respecto al último objetivo, examinar la relación entre satisfacción laboral con las variables sociodemográficas y laborales de los trabajadores, se observa una asociación positiva con la edad, con la antigüedad laboral y el tipo de contrato laboral.

Así, en referencia a la edad, trabajadores de más edad indican mayo satisfacción. En este sentido, la etapa de la adultez va a posibilitar el aprendizaje a la convivencia con determinadas situaciones y con ello, a la oportunidad de desarrollar la capacidad para hacer frente a las adversidades (Navarro et al., 2010). Por ello, podríamos presuponer que los trabajadores de mayor de edad han tenido más tiempo de adaptación y han establecido una relación más cercana y de superación a sus limitaciones tanto físicas como psicológicas que los trabajadores de menor edad. De la misma manera, se observa asociación entre satisfacción laboral y experiencia laboral. Si entendemos que la experiencia permite en numerosos casos, la adaptabilidad al puesto de trabajo, y asimismo, permite tener una visión más objetiva de matices que otros profesionales de menos experiencia, se podría entender que la antigüedad laboral favorezca este proceso de adaptación al desempeño profesional (Abrajan et al., 2009). Finalmente, en referencia al tipo de contrato, en los resultados se encuentra relación entre éste y la satisfacción laboral de los trabajadores, observándose asociación positiva entre la satisfacción laboral percibida por los trabajadores que tienen un contrato indefinido. Sin duda, la inseguridad laboral se ha convertido en la actualidad en una de las fuentes de estrés más significativas para muchos colectivos de trabajadores, siendo especialmente grave en España por las elevadas tasas de desempleo en este periodo de crisis económica (Riaño-Casallas et al., 2016).

A la luz de los resultados obtenidos en este estudio, se concluye que cuanto mayor empoderamiento adquieren los trabajadores con movilidad reducida, mayor capacidad poseen para hacer frente a sus adversidades y dificultades generadas debidas a las limitaciones del movimiento, lo que desemboca en una mayor satisfacción en todos los ámbitos de la vida y más concretamente en el ámbito laboral. A su vez, se refleja que algunas variables como la edad, experiencia laboral o tener un trabajo estable, son factores que repercuten favorablemente en el grado de satisfacción aboral de los trabajadores con discapacidad.

Sin embargo, el trabajo presenta algunas limitaciones que pueden influir en la magnitud de las asociaciones. La primera y más importante es el reducido número de personas que participaron en el estudio, condicionado sin duda por la escasa disponibilidad de la población de estudio en situación laboral activa accesible para la investigación de estudio. Asimismo, la selección de los participantes ha sido intencional, lo que en sentido estricto impide hablar de "muestra". Por otra parte, los participantes del estudio se ofrecieron voluntariamente para cumplimentar los cuestionarios, por lo que éstos, podrían tener diferentes motivaciones para participar. Estos aspectos pueden sobreestimar el nivel de empoderamiento y podrían sesgar la magnitud de algunas de las asociaciones detectadas.

No obstante, a pesar de la complejidad de salvar estas limitaciones, por la dificultad de acceder a una muestra más elevada, los resultados resultan interesantes, pues sugieren que el empoderamiento está relacionado con la satisfacción laboral en este colectivo. De esta forma, precisamente en este contexto donde el empoderamiento adquiere relevancia, no solo como elemento de superación, sino también como fenómeno en el que hay que profundizar, con el objetivo de que en cualquier programa de intervención se fomente la construcción y desarrollo de este constructo para incrementar la satisfacción laboral de los trabajadores que viven con el hándicap de tener movilidad reducida. 


\section{Referencias bibliográficas}

Abrajan, M. G. et al. (2009). "Grado de satisfacción laboral y condiciones de trabajo: una exploración cualitativa". Enseñanza e investigación en Psicología, 14(1), pp.105-118

Albrecht, G.L. et al. (2001). Handbook of Disability Studies, Thousand Oaks: Sage Publications.

Alcover de la Hera, C. M. et al. (2017). "Crisis económica, salud y bienestar en trabajadores con discapacidad". Revista de Psicología del Trabajo y de las Organizaciones, 33(2), pp.147-155.

Alcover de la Hera, C. M. y Pérez Torres, V. (2011). "Trabajadores con discapacidad: problemas, retos y principios de actuación en salud ocupacional”. Medicina y seguridad del trabajo, 57, pp. 206-223.

Baldridge, D. C. y Swift, M. L. (2013). "Withholding requests for disability accommodation: The role of individual differences and disability attributes". Journal of Management, 39, pp. 743-762.

Barnes, C. et al. (1997). Exploring disability. A sociological introduction. Cambridge: Polity Press.

Bejerholm, U. y Björkman, T. (2011). "Empowerment in supported employment research and practice: Is it relevant". International Journal of Social Psychiatry, 57(6), pp. 588-595.

Beehr, T.A. et al. (2000). "Work stressors and coworker support as predictors of individual strain and job performance". Journal of Organizational Behavior, 21(4), pp. 391-405.

Bertely, M. (2003). Educación, derechos sociales y equidad. México: COMIE.

Corrigan, P. (2002). "Empowerment and serious mental illness: treatment partners-hips and community opportunities”. Psychiatric Q, 73(3), pp. 217-228.

Corrigan, P. y Gelb, B. (2006). "Three programs that use mass approaches to challenge the stigma of mental illness". Psychiatric Services, 57(3), pp. 393-398.

Cuadra, A. y Veloso, C. (2007). "Liderazgo, clima y satisfacción laboral en las organizaciones". Universum Talca, 22(2), pp. 40-56.

Durá, A. y Salaberría, K. (2011). "Satisfacción laboral de los trabajadores con discapacidad”. Zerbitzuan, 49, pp.127-136.

Durán, M., et al. (2005). "Engagement y burnout en el ámbito docente: Análisis de sus relaciones con la satisfacción laboral y vital en una muestra de profesores". Revista de Psicología del Trabajo y de las Organizaciones, 21, pp. 1-2.

Eisenberger, R. et al. (2001). "Reciprocation of perceived organizational support”. Journal of Applied Psychology, 86(1), pp. 42-51.

Flores, N. et al. (2011). "Understanding quality of working life of workers with intellectual disabilities". Journal of Applied Research in Intellectual Disabilities, 24, pp.133-141.

Flores, N. et al. (2010). "Análisis de la calidad de vida laboral en trabajadores con discapacidad”. Zerbitzuan, 47, pp. 55-67.

Gallway, J. y Bernasek, A. (2004). "Literacy and women's empowerment in Indonesia: implications for policy". Journal of economic issues, 38(1), pp. 519-525. 
Hansen, J. B. y Nielsen, P. H. (2008). Handbook of heterogeneous catalysis. Ertl, G., Knözinger, H., Weikamp, J., Eds.

Judge, T. A. et al. (2012). "Job satisfaction and job affect”. The Oxford handbook of organizational psychology, 1, pp. 496-525.

Koberg, C. S. et al. (1999). "Antecedents and outcomes of empowerment: Empirical evidence from the health care industry". Group \& organization management, 24(1), pp. 71-91.

Kocman, A. y Weber, G. (2018). "Job Satisfaction, Quality of Work Life and Work Motivation in Employees with Intellectual Disability: a Sistematic-Review”. Journal of Applied Research in Intellectual Disabilities, 31, pp. 1-22.

Landa, J. M. et al. (2006). "Relación entre burnout e inteligencia emocional y su impacto en salud mental, bienestar y satisfacción laboral en profesionales de enfermería”. Ansiedad y estrés, 12, pp. 1-13.

Moore, M. E. et al. (2011). "The vocational well-being of workers with childhood onset of disability: Life satisfaction and perceived workplace discrimination". Journal of Vocational Behavior, 79, pp. 681-698.

Moreno, B. et al. (2009). "Consecuencias positivas y negativas en el trabajo: el rol de las expectativas laborales en el proceso de desgaste profesional”. Ciencia y trabajo 11(32), pp. 80-84.

Musitu, G. y Buelga, S. (2004). "Desarrollo comunitario y potenciación (empowerment)". En G. Musito et al. (eds.), Introducción a la psicología comunitaria (pp. 167-195). Barcelona: UOC.

Navarro-Pérez, J. J. y Martínez-Muñoz, E. V. (2014). "Trabajo Social Comunitario y Formación en Centros de Trabajo: una propuesta para la inserción de adolescentes problematizados desde la Educación Formal”. Comunitania: Revista Internacional de Trabajo Social y Ciencias Sociales, 8, pp. 87-104.

Navarro, E. et al. (2010). "Factores de satisfacción laboral evocados por los profesionales de la construcción en la Comunidad Valenciana (España)". Revista de la Construcción, 9(1), pp. 4-16.

OCDE (2010). Educating Teachers for Diversity:Meeting the Challenge. Paris: OCDE. Recuperado de http://www. oecd.org/document/38/0,3343,en_2649_35845581_44572006_1_1_1_1,00.html.

Organización Internacional del Trabajo (OIT) - UNESCO (2013). RBC con y para personas con discapacidad. Ponencia conjunta. Ginebra. Recuperado de https://www.ilo.org/global/research/global-reports/world-ofwork/2013/lang--es/index.htm.

Pecino-Medina, V. et al. (2015). "Clima y satisfacción laboral en el contexto universitario". Anales de psicología, 31(2), pp. 658-666.

Pallisera Diaz, M. y Rius Bonjoch, M. (2007). “¿Y después del trabajo, qué? Más allá de la integración laboral de las personas con discapacidad”. Revista de Educación, 342, pp. 329-348.

Peralta, A. C. et al. (2016). "Efecto de un programa de psicología positiva e inteligencia emocional sobre la satisfacción laboral y vital”. Salud \& Sociedad, 1(2), pp. 101-112.

Pérez Bilbao, J. y Fidalgo Vega, M. (1995). Escala general de Satisfacción Laboral NTP 394. Madrid: Ministerio de Trabajo y Asuntos Sociales, Instituto Nacional de Seguridad e Higiene en el Trabajo.

PNUD (1997). Informe sobre Desarrollo Humano 1997. Madrid: Mundi- Prensa.

Rappaport, J. (1984). "Studies in empowerment: Introduction to the issue". Prevention in Human Services, 3 , pp. 1-7. 
Riaño-Galán, A. et al. (2016). "Calidad de vida e inserción socio-laboral de jóvenes con discapacidad". Revista electrónica de investigación educativa, 18(1), pp. 112-127.

Riaño-Casallas, M. I. et al. (2016). "Evolución de un sistema de gestión de seguridad y salud en el trabajo e impacto en la accidentalidad laboral: Estudio de caso en empresas del sector petroquímico en Colombia". Ciencia \& Trabajo, 18(55), pp. 68-72.

Rico-Picó, J. et al. (2016). "Empowerment, satisfacción laboral e identificación organizacional en funcionarios andaluces". Reidocrea, monográfico, pp. 33-39.

Rhoades, L. y Eisenberger, R. (2002). "Perceived Organizational Support: A Review of the Literature”. Journal of Applied Psychology, 87(4), pp. 698-714.

Röger, U., et al. (2011). "Differences in individual empowerment outcomes of socially disadvantaged women: effects of mode of participation and structural changes in a physical activity promotion program". International Journal of Public Health, 56(5), pp. 465-473.

Rogers, E. S. et al. (1997). "A consumer-constructed scale to measure empowerment among users of mental health services". Psychiatric Service, 48(8), pp. 1042-1047.

Saavedra, E. y Villalta, M. (2008). "Estudio comparativo en Resiliencia con sujetos discapacitados motores". Revista Investigaciones en Educación, 8, pp. 81-90.

Sá-Fernandes, L. et al. (2018). "Empowerment promotion through competitive employment for people with psychiatric disabilities”. Journal of Vocational Rehabilitation, 49, pp. 259-263.

San Martín, R. y Pardo, A. (1989). Psicoestadística. Contrastes paramétricos y no paramétricos. Madrid: Pirámide.

Seibert, S. E. et al. (2011). "Antecedents and consequences of psychological and team empowerment in organizations: A meta-analytic review". Journal of Applied Psychology, 96(5), pp. 981-984.

Seibert, S. E. et al. (2004). "Taking empowerment to the next level: A multiple-level model of empowerment, performance, and satisfaction". Academy of Management Journal, 47(3), pp. 332-349.

Segado, S. (2011). Nuevas tendencias en Trabajo Social con Familias: una propuesta para la práctica desde el empowerment. Madrid: Trotta.

Suriá, R. S. (2017). "Relationship between resilience and empowerment in adults with reduced mobility". Quaderns de Psicologia, 19(3), pp. 253.

Veloso-Besio et al. (2015). "Capacitación en trabajadores: impacto de un programa, basado en psicología positiva y habilidades sociales, en satisfacción vital, satisfacción laboral y clima organizacional”. Interciencia, 40(11), pp. 736-746.

Wang, G. y Lee, P. D. (2009). "Psychological empowerment and job satisfaction: An analysis of interactive effects". Group \& Organization Management, 34(3), pp. 271-296.

Warr, P. et al. (1979). "Scales for the measurement of some work attitudes and aspects of psychological wellbeing". Journal of Occupational and Organizational Psychology, 52(2), pp.129-148.

Weissenberg, R. (1999). The politics of empowerment. Westport: Praeger.

Zimmerman, B. J. (2000). "Attaining self-regulation: A social cognitive perspective”. En M. Boekaerts et al. (eds.), Handbook of self-regulation (pp. 13-39). San Diego, Calif.: Academic Press. 